\title{
Hypothesis on the pathogenesis of vacuolar myelopathy, dementia, and peripheral neuropathy in AIDS
}

\author{
S V Tan, R J Guiloff
}

\begin{abstract}
Certain aspects of the clinical syndrome of dementia, cerebral atrophy, predominantly sensory neuropathy, and vacuolar myelopathy in AIDS resemble those seen in vitamin B12 deficiency. Pathologically, there are similarities not only in the changes in the spinal cord, but also in the brain and peripheral nerves. The pathogenesis of vacuolar myelopathy may be secondary to a combination of immune mediated myelin and oligodendrocyte injury, and simultaneous impairment of repair mechanisms due to a deficiency of S-adenosylmethionine (SAM). Products derived from macrophages may interfere directly with the methyl transfer cycle through the generation of reactive oxygen intermediates and reactions involving nitric oxide and peroxynitrite which may limit the supply of methionine for conversion to SAM, both by direct interaction as well as through inhibition of methionine synthase. Macrophage activation with secretion of cytokines and other biologically reactive substances within the nervous system is sustained in the late stages of HIV infection by the general effects of immune depletion, including loss of $T$ cells (with concomitant reduction of macrophage regulatory molecules) and recurrent opportunistic infections, and may be further augmented by the local presence of the virus itself (or its surface glycoprotein gp120). This would account for the common, but not exclusive, occurrence of vacuolar myelopathy in AIDS. The ability of the virus and its products to stimulate macrophage and microglial activation may also explain the association between severity ofvacuolar myelopathy and the presence of HIV encephalitis. A similar mechanism may underlie the pathogenesis of dementia, cerebral atrophy, and peripheral neuropathy. Local factors or differential susceptibility between the central and peripheral nervous system may determine whether myelinotoxic or neurotoxic processes predominate; the prominence of myelin involvement in the spinal cord, and axonal involvement peripherally may reflect both ends of this range, with the brain manifesting a more equal balance of both processes.

(F Neurol Neurosurg Psychiatry 1998;65:23-28)
\end{abstract}

Keywords: AIDS; myelopathy; peripheral neuropathy; dementia
Publications support an indirect relation between HIV and the pathogenesis of vacuolar myelopathy, peripheral neuropathy, and dementia. The role of products and cytokines derived from macrophages or microglia has been emphasised by some, ${ }^{1-4}$ and defective methylation due to a deficiency of S-adenosylmethionine (SAM) by others, ${ }^{5-8}$ with some suggesting that the two may be linked. ${ }^{5}$

We propose that concomitant SAM depletion and cytokine or oxygen mediated toxicity underlie myelin and neuronal damage in AIDS. The SAM depletion may occur as a result of (a) reduced SAM production secondary to limitation in methionine availability due to reduced methionine production caused by inhibition of methionine synthase by macrophage derived nitric oxide (NO), and oxidation of methionine by peroxynitrite, and (b) increased consumption of SAM in repair mechanisms triggered by the toxic effects of cytokines and oxygen radicals on myelin or membranes.

This model suggests a direct link between macrophage activation and SAM depletion in AIDS and explains the neuroanatomical lesions in vacuolar myelopathy, which is pathologically indistinguishable from subacute combined degeneration of the cord (SACD)..${ }^{90} \mathrm{~A}$ similar clinicopathological syndrome of dementia, vacuolar myelopathy, peripheral neuropathy, and SAM depletion is seen in vitamin B12 deficiency.

The proposed pathogenesis is discussed in the following order: vacuolar myelopathy, dementia, and peripheral neuropathy. Figure 1 shows a summary of the pathogenetic cascade. Figure 2 shows the relation of methionine, vitamin B12, and folate with SAM production and the association of the single carbon transfer pathway with glutathione metabolism.

Vacuolar myelopathy

(A) ACTIVATION OF MACROPHAGES, MONOCYTES, OR MICROGLIA

The secretion of immunoactive or toxic substances by activated macrophages and microglia contributes to CNS immunopathology in AIDS. ${ }^{12}{ }^{11}$ Factors which may contribute to macrophage activation within the CNS in AIDS (figure 1) include: $(a)$ decreased production of macrophage regulatory lymphokines interleukin-4 (IL-4) and IL-10 $0^{2}$; (b) recurrent infections; (c) increasing viral load and interaction of gp120 with macrophages leading to cytokine interleukin 1 (IL-1) and arachidonic acid metabolite production ${ }^{12} ;(d)$ 


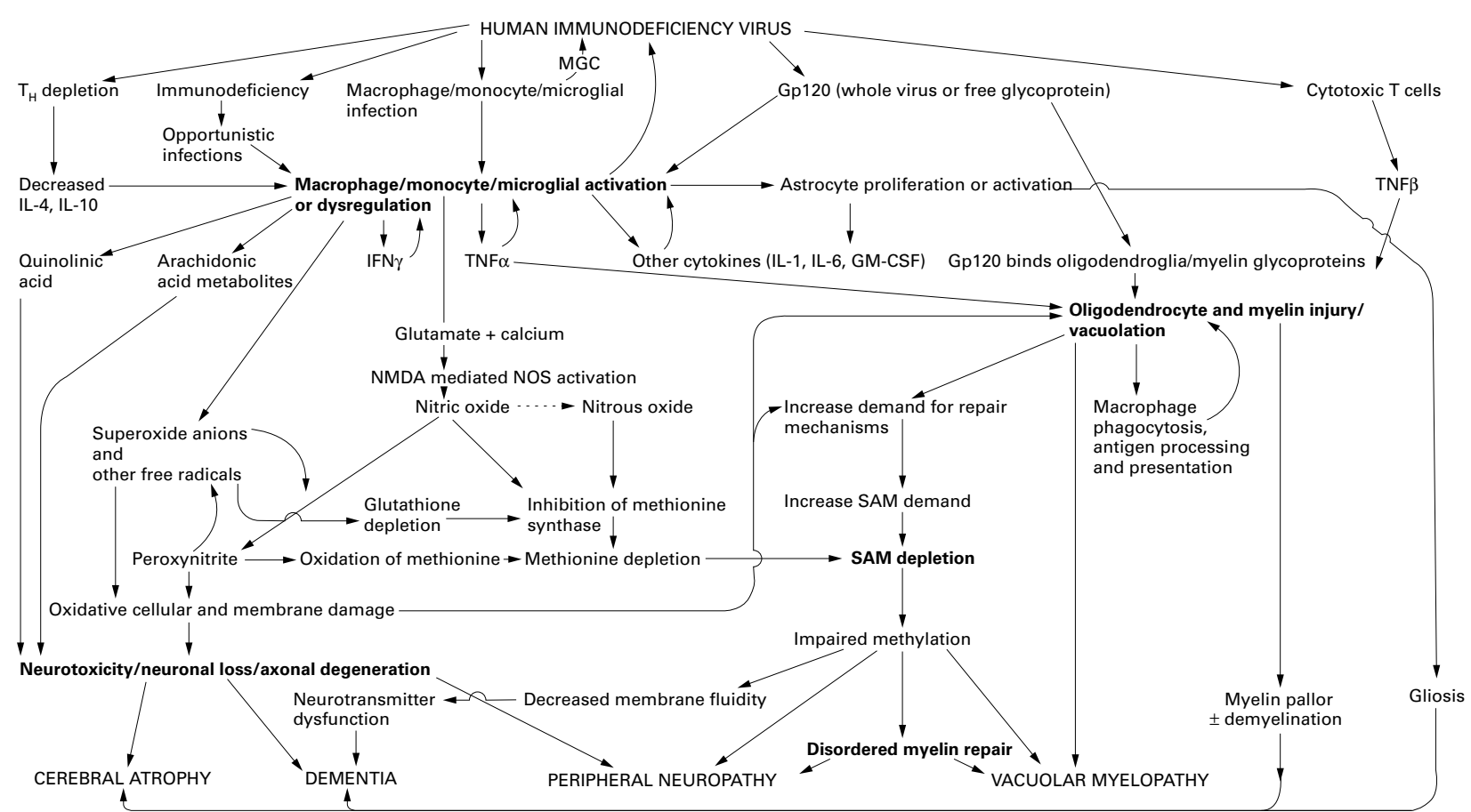

Figure 1 Hypothesis on the pathogenesis of vacuolar myelopathy, dementia, cerebral atrophy, and peripheral neuropathy in HIV disease. A schematic representation.

secretion of tumour necrosis factor- $\alpha(\mathrm{TNF} \alpha)$ (also stimulated by IL-1). ${ }^{13}$ TNF $\alpha$ up regulates the transcription of integrated HIV, ${ }^{14}{ }^{15}$ leading to a positive feedback cycle of $\mathrm{TNF} \alpha$ production, HIV replication, and macrophage activation (fig 1).

In vacuolar myelopathy, macrophages may be attracted to specific areas of cord by degenerating myelin (see section (c)on SAM depletion). If HIV by itself does not cause the changes found in vacuolar myelopathy, but when present within macrophages, augments their capacity to secrete myelinotoxic substances, several conflicting findings may be clarified; for instance, the apparent association between the presence of HIV infected macrophages and the severity of vacuolar change, ${ }^{16}$ the occurrence of vacuolar myelopathy in the absence of detectable HIV in the cord, the lack of correlation between the presence of coincidental HIV myelitis and the severity of vacuolar myelopathy, ${ }^{17} 18$ and the frequent coexistence of HIV encephalitis (HIVE) and vacuolar myelopathy in AIDS. ${ }^{19}$

Astrogliosis in the brain in dementia and in cords with and without vacuolar myelopathy is common in AIDS. Macrophage-astroglial interactions are an important source of immunopathology. ${ }^{20}$ Coactivation of astrocytes by $\mathrm{TNF} \alpha$ results in the secretion of other cytokines such as IL-6 and granulocytemacrophage colony stimulating factor (GMCSF), which activate macrophages and microglia ${ }^{21}$ and which act synergistically with $\mathrm{TNF} \alpha$ to induce HIV-1 expression in macrophages. ${ }^{22}$

(B) OLIGODENDROCYTE AND MYELIN INJURY AND VACUOLATION

The main pathological insult in vacuolar myelopathy is to myelin; axonal degeneration occurs secondarily in severe cases. ${ }^{9}$ Tumour necrosis factor- $\alpha$ may play a major part in oligodendrocyte and myelin injury in vacuolar myelopathy and in the CNS in AIDS. It is produced by activated macrophages, microglia, astrocytes, and endothelial cells and causes myelin vacuolation of mouse spinal cord oligodendrocytes in vitro. ${ }^{23}$ The presence of $\mathrm{TNF} \alpha$ within macrophages and microglia in the lateral and posterior columns even in mild, presumably early, vacuolar myelopathy ${ }^{4}$ is consistent with its direct role in the pathogenesis of myelin vacuolation in vacuolar myelopathy.

The viral surface glycoprotein gp120 may bind to oligodendroglia, ${ }^{24}$ and to peripheral myelin associated glycoprotein. ${ }^{25}$ Infected macrophages expressing gp120 may similarly bind to, and damage, such glycoproteins on oligodendrocytes and central myelin. Alternatively, gp120 bound to myelin glycoproteins may be ingested by scavenging macrophages attracted by damaged myelin. Macrophages may act as antigen presenting cells, thus further enhancing the immune reaction, in conjunction with $\mathrm{TNF} \alpha$ mediated upregulation of MHC class II antigen expression (fig 1). ${ }^{26} \mathrm{Oli}-$ godendrocytes in transgenic mice with a vacuolar myelopathy express HIV-1 proteins. ${ }^{27}$

The generation of reactive oxygen intermediates and other reactive species-such as NO and peroxynitrite-by activated macrophages and microglia may damage myelin and membranes. Nitric oxide production is stimulated by many substances including $\mathrm{TNF} \alpha,{ }^{28}$ interferon- $\gamma \quad($ IFN $\gamma),{ }^{29}$ lipopolysaccharide, ${ }^{29}$ IL- $1,{ }^{30}$ interactions with astroglial cells, ${ }^{20}$ and perhaps arachidonic acid. ${ }^{31}$

Peroxynitrite, a powerful oxidant formed by combination of $\mathrm{NO}$ with the superoxide free radical, can attack a wide range of biological targets including thiols, ${ }^{32}$ lipids, ${ }^{33}$ methionine, ${ }^{34}$ 


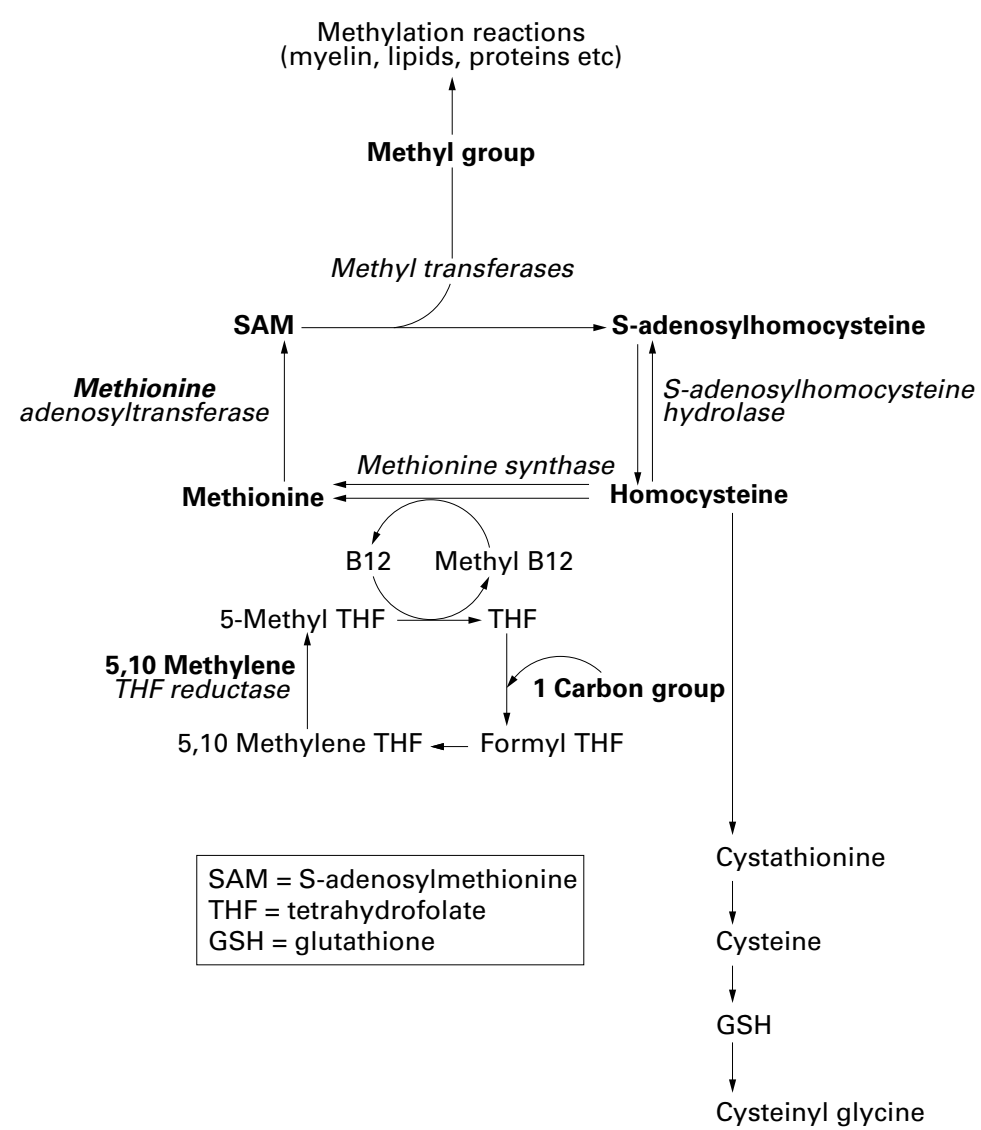

Figure 2 The single carbon (methyl) transfer pathway. Relation with B12 and glutathione metabolism. (Modified from Surtees 1993, Bottiglieri et al 1994, and Castagna et al 1995..$^{710}$ ) the biological methylations that modify proteins (including myelin basic protein), nucleic acids, fatty acids, porphyrins, phospholipids, polysaccharides, and biogenic amines such as catecholamines. ${ }^{10}{ }^{39}$ A SAM deficiency is critical to the development of demyelination in cobalamin (vitamin B12) deficiency. ${ }^{10}{ }^{40}$ It may also be essential to the pathogenesis of vacuolar myelopathy. ${ }^{5}$

Comparisons of CSF concentrations of the components of the methyl transfer pathway in HIV seropositive patients with neurological disease and HIV seronegative controls show decreased SAM, ${ }^{568}$ increased S-adenosylhomocysteine (SAH) and reduced $\mathrm{SAM} / \mathrm{SAH}$ (methylation) ratio, ${ }^{6}$ normal homocysteine, ${ }^{8}$ and normal or occasionally low methionine (fig 2). ${ }^{5}$ Decreased concentrations of folate in CSF were found by some authors ${ }^{5}$ but not by others. ${ }^{8}$ Studies of the components of the trans-sulphuration pathway, showed decreased concentrations of glutathione and cysteinyl glycine but normal concentrations of cysteine in CSF (fig 2). ${ }^{8}$

The low concentrations of SAM in patients with HIV with neurological disease may be caused by excessive consumption of SAM in methylation reactions for repair of immunological damage to the CNS. ${ }^{5}$ The reasons for the apparent inability of the methyl transfer cycle to generate sufficient SAM are unknown. The linear relation between SAM and methionine concentrations in CSF in patients with HIV but not in controls suggests that the availability of methionine may be limiting the production of SAM. ${ }^{5}$ The reasons for limitation of methionine availability are unclear. A deficiency of 5-methyltetrahydrofolate induced by macrophage secreted neopterin has been suggested, ${ }^{5}$ but there is no evidence of folate deficiency in patients with HIV with neurological complications (including myelopathy). ${ }^{8}$

The supply of methionine is determined by the activity of the vitamin B12 dependent methionine synthase, acting on homocysteine (fig 2). The diet ${ }^{41}$ is unlikely to be an adequate source, as the neurological consequences of SAM deficiency occur when methionine synthase is inhibited by B12 or folate deficiency or nitrous oxide misuse. ${ }^{42} 43$

Vitamin B12 deficiency may exacerbate SAM deficiency in some patients, but it cannot be the primary cause. Firstly, most patients with vacuolar myelopathy do not have B12 deficiency. ${ }^{918}$ Secondly, although B12 deficiency is common in AIDS, ${ }^{44}$ those shown to be SAM deficient were B12 and folate replete. ${ }^{6} 8$

Limitation in the bioavailability of methionine may relate to the production of $\mathrm{NO}$ and peroxynitrite by activated macrophages and microglia. Peroxynitrite oxidises methionine to ethylene or methionine sulphoxide. ${ }^{34}$ This powerful oxidant may be generated in the vicinity of activated macrophages which are capable of secreting NO and superoxide simultaneously, and is likely to cause local reduction in methionine concentrations.

Bovine heart cytochrome c oxidase catalyses the reduction of $\mathrm{NO}$ to nitrous oxide in 
anaerobic conditions, ${ }^{45}$ raising the further possibility that NO derived from macrophages gives rise to nitrous oxide in vivo with consequent methionine synthase inhibition and neurological sequelae - such as SACD - as seen in nitrous oxide misuse. ${ }^{42}{ }^{43}$ Also, NO itself may cause a rapid dose dependent inhibition of methionine synthase. ${ }^{46}$ Reduced CNS concentrations of glutathione in patients with HIV may further contribute to impairment of methionine synthase activity. ${ }^{8} 47$

Methionine synthase inhibition in isolation normally causes a rise in homocysteine concentrations, but this was not found. ${ }^{8}$ Homocysteine concentrations may be kept within normal limits by several mechanisms. Firstly, with the production of oxygen radicals by macrophages in AIDS, homocysteine may be consumed by the trans-sulphuration pathway to replenish falling concentrations of the antioxidant glutathione, which was found to be low in these patients (fig 2). ${ }^{8}$ Secondly, the kinetics of the enzyme SAH hydrolase (fig 2) favour the formation of SAH, the reaction in vivo being normally driven in the opposite direction (of homocysteine) only by the rapid removal of homocysteine and adenine. ${ }^{10}$ Normal or rising homocysteine concentrations would therefore be expected to favour the conversion of homocysteine to SAH. This may also account for the high concentrations of SAH and low methylation ratio. ${ }^{6}$

Thus, in AIDS, macrophage derived products in the CNS may lead to increased consumption of SAM, and simultaneously limit its production. Where SAM deficiency is sustained chronically, this may lead to the clinical and pathological changes of vacuolar myelopathy. Such a link between macrophage activation and SAM metabolism may account for the high incidence of vacuolar myelopathy in AIDS, as well as its occurrence in immunosuppressed HIV seronegative people. ${ }^{48}$

\section{Dementia and cerebral atrophy}

The brains of patients with AIDS with dementia or cerebral atrophy show varying degrees of demyelination, neuronal loss, gliosis, microglial activation, multinucleated giant cells, and detectable HIV antigen or RNA. ${ }^{49} 50$ Probable mechanisms responsible for the non-specific neuropathological changes are as follows: $(a)$ the gliosis results from astrocyte proliferation in response to $\mathrm{TNF}^{51} ;(b)$ the myelin pallor may be due to SAM deficiency or the production of $\mathrm{TNF} \alpha$ or other cytokines and oxygen radicals ${ }^{1152}$; and $(c)$ the neuronal $\operatorname{loss}^{53}$ may be due to local production of neurotoxins such as quinolinic acid, $^{54}$ arachidonic acid metabolites, ${ }^{20}$ free radicals, and $\mathrm{NO}^{28} \mathrm{NO}$, produced by certain types of neurons ${ }^{36}$ as well as macrophages, microglia, and astrocytes, may also mediate the neurotoxicity of gp $120 . .^{55}$

The presence and degree of dementia and/or cerebral atrophy cannot be correlated specifically with any one neuropathological feature. ${ }^{350}$ Several explanations could be forwarded.

Firstly, the neurological features may be the product of variably contributing factors, in each person (fig 1). Gliosis, myelin pallor, or neuronal loss may be more prominent in some patients, whereas in others multinucleated giant cells and microglial nodules may predominate if the level of productive viral infection is high. The degree of SAM deficiency may contribute to the extent of demyelination. In B12 deficiency, demyelination may vary from small, often perivascular, foci to dissemination over wide areas of the corona radiata, sometimes accompanied by diffuse gliosis, ${ }^{56}$ closely resembling descriptions in AIDS. $^{57}$

Secondly, impairment of neurotransmitter function (as in SAM deficiency), which may contribute to AIDS dementia, is not detectable by routine histopathological techniques. The SAM dependent enzymatic methylation of phospholipids plays an important part in the transduction of receptor mediated signals through cellular membranes. Catecholamine neurotransmitters interacting with cell surface receptors initiate a cascade of biochemical and physical changes in local domains of the membrane leading to increased phospholipid methylation and mobility of receptors. ${ }^{58}$ Concentrations of SAM in the CSF also seem to influence brain monoamine metabolism. ${ }^{59}$

\section{Peripheral neuropathy}

A distal symmetric predominantly sensory axonal neuropathy (DSPN) of unknown aetiology is common in AIDS. Some authors distinguish between the painful and non-painful subgroups, ${ }^{60}$ whereas others do not. ${ }^{61}$ In HIV disease, as in HIV seronegative people, both painful and non-painful distal neuropathies are heterogeneous entities. A proportion of the painful subgroup may be caused by cytomegalovirus (CMV) ${ }^{60}{ }^{62}{ }^{63}$; other causes include vasculitis, ${ }^{64}$ drugs, ${ }^{65}$ inflammatory processes, ${ }^{66}$ nutritional deficiencies, and diabetes. However, in most patients with DSPN, no cause is found. Macrophage mediated immunopathology, together with SAM depletion, may be important factors in the pathophysiology of DSPN in AIDS. The mechanism of macrophage recruitment in peripheral neuropathy may be similar to that described for vacuolar myelopathy.

Vitamin B12 deficiency may present as a painful, or non-painful neuropathy. ${ }^{67}{ }^{68}$ There is no correlation between serum B12 concentrations and the presence of distal sensory peripheral neuropathy in AIDS. ${ }^{69}$ As described in vacuolar myelopathy, SAM deficiency can occur with normal serum concentrations of B12 and folate and may contribute to the pathophysiology of DSPN.

Wallerian-like degeneration of myelinated and unmyelinated fibres, and distal axonal degeneration involving both the peripheral and central branches occur in DSPN. ${ }^{60}{ }^{61}{ }^{70}$ Axonal atrophy seems to be found in painful but not in non-painful peripheral neuropathy. ${ }^{70}$ The pathology of peripheral neuropathy in B12 deficiency is poorly documented but a similar reduction in numbers of myelin sheaths and axons, with degeneration in the centrally directed posterior root fibres, has been 
described. $^{71}$ In $\mathrm{B} 12$ deficiency in monkeys, both Wallerian type axonal and myelin degeneration were seen in the peripheral nerves. ${ }^{72}$

Macrophages expressing $\mathrm{TNF} \alpha$ within degenerating nerve fibres seem to be activated to a greater extent, and present in higher density for a given degree of Wallerian-like degeneration in AIDS, compared with seronegative controls with neuropathy. ${ }^{61}$ Cytokines secreted by activated macrophages may account for the damage to unmyelinated and myelinated fibres.

\section{Clinical implications}

Supplementation with SAM has been used clinically in the treatment of various disorders including depression ${ }^{7}$ and osteoarthritis. ${ }^{73}$ Concentrations of SAM in the CSF can be raised by systemic oral SAM supplementation. ${ }^{80}$ By contrast with methionine, it seems to be relatively non-toxic, both in short and long term animal studies and in over 10 years of clinical use. ${ }^{74}$ Also, there has been recent in vitro evidence that SAM may protect transplanted hepatocytes from the toxic effects of cytokines, ${ }^{75}$ prevent glutamate cytotoxicity in cultured cortical neurons, ${ }^{76}$ and raise glutathione concentrations in $\mathrm{CSF}^{8}$ If this hypothesis is correct, SAM supplementation early in HIV disease may significantly reduce the incidence of vacuolar myelopathy, dementia, and peripheral neuropathy.

1 Tyor WR, Glass JD, Baumrind N, et al. Cytokine expression of macrophages in HIV-1-associated vacuolar myelopathy. Neurology 1993;43:1002-9.

2 Wesselingh SL, Glass J, McArthur JC, et al. Cytokine dysregulation in HIV-associated neurological disease. $A d v$ Neuroimmunol 1994;4:199-206.

3 Tyor WR, Wesselingh SL, Griffin JL, et al. Unifying hypothesis for the pathogenesis of HIV-associated dementia complex, vacuolar myelopathy, and sensory neuropathy. I Acquir Immune Defic Syndr Hum Retrovirol 1995;9:379-88.

4 Tan SV, Guiloff RJ, Henderson DC, et al. AIDS-associated vacuolar myelopathy and tum

5 Surtees R, Hyland K, Smith I. Central-nervous-system methyl-group metabolism in children with neurological methyl-group metabolism in children with neurological
complications of HIV infection. Lancet 1990;335:619-21.

6 Keating JN, Trimble KC, Mulcahy F, et al. Evidence of brain methyltransferase inhibition and early brain involvebrain methyltransferase inhibition and early brain invo

7 Bottiglieri T, Hyland K, Reynolds EH. The clinical potential of ademetionine (S-adenosylmethionine) in neurological disorders. Drugs 1994;48:137-52.

8 Castagna A, Le Grazie C, Accordini A, et al. Cerebrospinal fluid S-adenosylmethionine (SAMe) and glutathione concentrations in HIV infection: effect of parenteral treatmen with SAMe. Neurology 1995;45:1678-83.

9 Petito CK, Navia BA, Cho ES, et al. Vacuolar myelopathy pathologically resembling subacute combined degeneration in patients with the acquired immunodeficiency syndrome. N Engl f Med 1985;312:874-9.

10 Surtees R. Biochemical pathogenesis of subacute combined degeneration of the spinal cord and brain. F Inherit Metab Dis 1993;16:762-70.

11 Tyor WR, Glass JD, Griffin JW, et al. Cytokine expression in the brain during the acquired immunodeficiency syn-

drome. Ann Neurol 1992;31:349-60.
12 Wahl LM, Corcoran ML, Pyle SW, et al. Human immunodeficiency virus glycoprotein (gp120) induction of monocyte arachidonic acid metabolites and interleukin 1. Proc Natl Acad Sci (USA) 1989;86:621-5.

13 Benveniste EN. Cytokine circuits in the brain: implications for AIDS dementia complex. In: Price RW, Perry SW, eds HIV, AIDS and the brain. New York: Raven Press, 1994;72: $71-88$

14 Poli G, Kinter A, Justement JS, et al. Tumor necrosis factor $\alpha$ functions in an autocrine manner in the induction of human immunodeficiency virus expression. Proc Natl Acad Sci (USA) 1990;87:782-5.

15 Rosenberg ZF, Fauci AS. Cytokine induction of HIV expression. Immunol Today 1990;11:176-80.

16 Weiser B, Peress N, La Neve D, et al. Human immunodeficiency virus type 1 expression in the central nervous system correlates directly with extent of disease. Proc Natl Acad Sci USA 1990;87:3997-4001.

17 Rosenblum M, Scheck AC, Cronin K, et al. Dissociation of AIDS-related vacuolar myelopathy and productive HIV-1 infection of the spinal cord. Neurology 1989;39:892-6.
18 Tan SV, Guiloff RJ, Scaravilli F. AIDS-associated vacuolar myelopathy - a morphometric study. Brain 1995;118:124761

19 Petito CK, Vecchio D, Chen Y-T. HIV antigen and DNA in AIDS spinal cords correlate with macrophage infiltration but not with vacuolar myelopathy. F Neuropathol Exp Neurol 1994;53:86-94

20 Genis P, Jett M, Bernton EW, et al. Cytokines and arachidonic metabolites produced during human immunodeficiency virus (HIV)-infected macrophage-astroglia
interactions: implications for the neuropathogenesis of HIV disease. F Exp Med 1992;176:1703-18.

21 Twerdy DJ, Glazer EW, Mott PL, et al. Modulation by tumor necrosis factor- $\alpha$ of human astroglial cell production of granulocyte-macrophage colony-stimulation factor (GM-CSF) and granulocyte colony-stimulationg factor (G-CSF). F Neuroimmunol 1991;32:269-78.

22 Rosenberg ZF, Fauci AS. Immunopathogenesis of HIV infection. FASEB f 1991;5:2382-90.

23 Selmaj KW, Raine CS. Tumor necrosis factor mediates myelin and oligodendrocyte damage in vitro. Ann Neurol 1988;23:339-46

24 Apostolski S, McAlarney T, Quattrini A, et al. The gp120 glycoprotein of human immunodeficiency virus type 1 binds to sensory ganglion neurons. Ann Neurol 1993;34: 855-63.

25 van den Berg LH, Sadiq SA, Lederman S, et al. The gp120 glycoprotein of HIV-1 binds to sulfatide and to the myelin associated glycoprotein. F Neurosci Res 1992;33:513-8.

26 Sherry B, Cerami A. Cachectin/tumor necrosis factor exerts endocrine, paracrine, and autocrine control of inflammatory responses. $\mathcal{f}$ Cell Biol 1988;107:1269-77.

27 Goudreau G, Carpenter S, Beaulieu N, et al. Vacuolar myelopathy in transgenic mice expressing human immunodeficiency virus type 1 proteins under the regulation of the myelin basic protein promoter. Nat Med 1996;2:655-61.

28 Bukrinsky MI, Nottet HSLM, Schmidtmayerova H, et al. Regulation of nitric oxide synthase activity in human immunodeficiency virus type 1 (HIV-1)-infected monocytes: implications for HIV-associated neurological disease. F Exp Med 1995;181:735-45.

29 Stuehr DJ, Cho HJ, Kwon NS, et al. Purification and characterization of the cytokine-induced macrophage nitric oxide synthase: an FAD- and FMN-containing flavoprotein. Proc Natl Acad Sci (USA) 1991;88:7773-7.

$30 \mathrm{McC}$ all T, Vallence P. Nitric oxide takes centre-stage with newly defined roles. Trends Pharmacol Sci 1992;13:1-6.

31 Miller B, Sarantis M, Traynelis SF, et al. Potentiation of NMDA receptor currents by arachidonic acid. Nature 1992;355:722-5.

32 Radi R, Beckman JS, Bush KM, et al. Peroxynitrite-induced membrane lipid peroxidation: the cytotoxic potential of superoxide and nitric oxide. Arch Biochem Biophys 1991; 288:481-7.

33 Radi R, Beckman JS, Bush KM, et al. Peroxynitrite oxidation of sulfhydryls. The cytotoxic potential of superoxide and nitric oxide. F Biol Chem 1991;266:424450

34 Pryor WA, Jin X, Squadrito GL. One- and two-electron oxidations of methionine by peroxynitrite. Proc Natl Acad Sci (USA) 1994;91:11173-7.

35 Olanow CW. A radical hypothesis for neurodegeneration. Trends Neurosci 1993;16:439-44.

36 Snyder SH. Nitric oxide and neurons. Curr Opin Neurobiol 1992;2:323-7.

37 Selmaj K, Raine CS, Farooq M, et al. Cytokine cytotoxicity against oligodendrocytes: apoptosis induced by lymphoagainst oligodendrocytes: apoptos

38 Chiodi F, Norkrans F, Hagberg L, et al. Human immunodeficiency virus infection of the brain. II Detection of intrathecally synthesized antibodies by enzyme linked immunosorbent assay and imprint immunofixation. 7 Neurol Sci 1988;87:37-48.

39 Baldessarini RJ. Neuropharmacology of S-adenosyl-Lmethionine. Am f Med 1987 83(suppl 5A):95-103.

40 Surtees R, Leonard J, Austin S. Association of demyelination with deficiency of cerebrospinal-fluid $\mathrm{S}$-adenosylmethionine in inborn errors of methyl-transfer pathway. Lancet 1991;338:1550-4.

41 Scott JM, Weir DG. The methyl folate trap. Lancet $1981 ; \mathrm{i}: 337-40$.

42 Dinn JJ, McCann S, Wilson P, et al. Animal model for subacute combined degeneration. Lancet:1978;ii:1154.

43 Layser RB. Myeloneuropathy after prolonged exposure to nayser RB. Myeloneuropathy after pro
nitrous oxide. Lancet 1978;ii:1227-30.

44 Harriman GR, Smith PD, Horne MK, et al. Vitamin B12 malabsorption in patients with acquired immunodeficiency malabsorption in patients with acquired immun
syndrome. Arch Intern Med 1989;149:2039-41.

45 Zhao XJ, Sampath J, Caughey WS. Cytochrome c oxidase catalysis of the reduction of nitric oxide to nitrous oxide. Biochem Biophys Res Commun 1995;212:1054-60.

46 Nicolaou A, Ast T, Garcia CV, et al. In vitro $\mathrm{NO}$ and $\mathrm{N}_{2} \mathrm{O}$ inhibition of the branch point enzyme vitamin $\mathrm{B} 12$ dependent methionine synthase from rat brain synaptosomes. Biochem Soc Trans 1994;22:296S.

47 Pajares M, Duran C, Corrales F, et al. Modulation of rat liver S-adenosylmethionine synthetase activity by glutathione. F Biol Chem 1992;367:17598-605.

48 Kamin SS, Petito CK. Idiopathic myelopathies with white matter vacuolation in non-acquired immunodeficiency matter vacuolation in non-acquired immunod

49 Gelman BB, Guinto FC Jr. Morphometry, histopathology, and tomography of cerebral atrophy in the acquired immunodeficiency syndrome. Ann Neurol 1992;31:32-40. 
50 Glass JD, Wesselingh SL, Selnes O, et al. Clinicalneuropathological correlations in HIV-associated demenneuropathological correlations
tia. Neurology 1993;43:2230-7.

51 Lachman LB, Brown DC, Dinarello CA. Growth promoting effect of recombinant interleukin-1 and tumor necrosis factor for a human astrocytoma cell line. F Immunol 1987;144 129-35.

52 Wesselingh SL, Power C, Glass JD, et al. Intracerebral cytokine messenger RNA expression in acquired immunodeficiency syndrome dementia. Ann Neurol 1993;33:57682 .

53 Everall IP, Luthert PJ, Lantos PL. Neuronal loss in the frontal cortex in HIV infection. Lancet 1991;337:1119-21.

54 Heyes MP, Brew BJ, Martin A, et al. Quinolinic acid in cerebrospinal fluid and serum in HIV-1 infection: relationship to clinical and neurological status. Ann Neurol 1991;29: 202-9.

55 Dawson VL, Dawson TM, Uhl GR, et al. Human immunodeficiency virus type 1 coat protein neurotoxicity mediated by nitric oxide in primary cortical cultures. Proc Natl Acad by nitric oxide in primary cort

56 Duchen LW, Jacobs JM. Nutritional deficiencies and metabolic disorders. In: Adams JH, Duchen LW, eds. Greenfield' neuropathology. 5th ed. London: Edward Arnold, 1992 811-80.

57 Schmidbauer M, Huemer M, Cristina S, et al. Morphological spectrum, distribution, and clinical correlation of white matter lesions in AIDS brains. Neuropathol App Neurobiol 1992;18:489-501.

58 Hirata F, Axelrod J. Phospholipid methylation and biological signal transmission. Science 1980;209:1082-90.

59 Bottiglieri T, Laundy M, Martin R, et al. $\mathrm{S}$-adenosylmethionine influences monoamine metabolism. Lancet $1984 ; \mathrm{i} \cdot 224$

60 Fuller GN, Jacobs JM, Guiloff RJ. Nature and incidence of peripheral nerve syndromes in HIV infection. I Neurol Neurosurg Psychiatry 1993;56:372-81.

61 Griffin JW, Wesselingh SL, Griffin DE, et al. Peripheral nerve disorders in HIV infection: similarities and contrasts nerve disorders in HIV infection: similarities and contrasts with central nervous system disorders. In: Price RW, Perry 1994;72:159-82.

62 Fuller GN, Jacobs JM, Guiloff RJ. Association of painful peripheral neuropathy in AIDS with cytomegalovirus infection. Lancet 1989;ii:937-41.

63 Mastroianni CM, Sebastiani G, Folgori F, et al. Detection of cytomegalovirus-matrix protein (pp65) in leukocytes of
HIV-infected patients with painful peripheral neuropathy. $\mathcal{F}$ Med Virol 1994;44:172-5.

64 Chamouard JM, Smadja D, Chaunu MP, et al. Neuropathie par vasculite nécrosante au cours de l'infection par le VIH1 Neuropathy caused by necrotizing vasculitis in HIV-1 infection). Rev Neurol 1993;149:358-61.

65 Simpson DM, Tagliati M. Nucleoside analogue-associated peripheral neuropathy in human immunodeficiency virus peripheral neuropathy in human immunodeficiency virus 1995;9:153-61.

66 Lipkin WI, Parry G, Kiprov D, et al. Inflammatory neuropathy in homosexual men with lymphadenopathy. Neurology 1985;35:1479-83.

67 Victor M, Lear AA. Subacute combined degeneration of the spinal cord. Current concepts of the disease process. Value of serum vitamin B12 determinations in clarifying some of of serum vitamin $\mathrm{B} 12$ determinations in clarifying some of

68 Healton EB, Savage DG, Brust JCM, et al. Neurologic aspects of cobalamin deficiency. Medicine 1991;70:229-45.

69 Veilleux M, Paltiel O, Falutz J. Sensorimotor neuropathy and abnormal vitamin B12 metabolism in early HIV infection. Can F Neurol Sci 1995;22:43-6.

70 Fuller GN, Jacobs JM, Guiloff RJ. Axonal atrophy in the painful peripheral neuropathy of AIDS. Acta Neuropathol (Berl) 1990;81:198-203.

71 Foster D B. Degeneration of peripheral nerves in pernicious anemia. Archives of Neurology and Psychiatry 1945;54:1029.

72 Scott JM, Dinn J, Wilson P, et al. Subacute combined degeneration. Lancet 1982;i:51-2.

73 Berger R, Nowak H. A new medical approach to the treatment of osteoarthritis. Report of an open phase IV study with ademetionine. Am $\mathcal{f}$ Med 1987;83(suppl $5 \mathrm{~A}): 84-8$.

74 Stramentinoli G. Pharmacologic aspects of S-adenosylmethionine. Am f Med 1987;83(suppl 5A):35-

75 Vara E, Arias-Diaz J, Garcia C, et al. S-adenosyl-methionine may protect transplanted hepatocytes against the toxic effects of cytokines. Transplant Proc 1994;26:3364-6.

76 Akaike A, Tamura Y, Sato Y, et al. Protective effects of a vitamin B12 analog, methylcobalamin, against glutamate cytotoxicity in cultured cortical neurons. Eur 7 Pharmacol $1993 ; 241: 1-6$ 